TRANSACTIONS OF THE

AMERICAN MATHEMATICAL SOCIETY

Volume 337, Number 1, May 1993

\title{
ALGEBRAIC CYCLES AND APPROXIMATION THEOREMS IN REAL ALGEBRAIC GEOMETRY
}

\author{
J. BOCHNAK AND W. KUCHARZ
}

Dedicated to the memory of Mario Raimondo

\begin{abstract}
Let $M$ be a compact $C^{\infty}$ manifold. A theorem of Nash-Tognoli asserts that $M$ has an algebraic model, that is, $M$ is diffeomorphic to a nonsingular real algebraic set $X$. Let $H_{\mathrm{alg}}^{k}(X, \mathbb{Z} / 2)$ denote the subgroup of $H^{k}(X, \mathbb{Z} / 2)$ of the cohomology classes determined by algebraic cycles of codimension $k$ on $X$. Assuming that $M$ is connected, orientable and $\operatorname{dim} M \geq$ 5 , we prove in this paper that a subgroup $G$ of $H^{2}(M, \mathbb{Z} / 2)$ is isomorphic to $H_{\mathrm{alg}}^{2}(X, \mathbb{Z} / 2)$ for some algebraic model $X$ of $M$ if and only if $w_{2}(T M)$ is in $G$ and each element of $G$ is of the form $w_{2}(\xi)$ for some real vector bundle $\xi$ over $M$, where $w_{2}$ stands for the second Stiefel-Whitney class. A result of this type was previously known for subgroups $G$ of $H^{1}(M, \mathbb{Z} / 2)$.
\end{abstract}

\section{INTRODUCTION AND STATEMENT OF THE MAIN RESULTS}

A $C^{\infty}$ submanifold $M$ of $\mathbb{R}^{n}$ is said to admit an algebraic approximation if there exists a $C^{\infty}$ embedding $e: M \rightarrow \mathbb{R}^{n}$, arbitrarily close in the $C^{\infty}$ topology to the inclusion mapping $M \hookrightarrow \mathbb{R}^{n}$, such that $X=e(M)$ is a nonsingular algebraic subset of $\mathbb{R}^{n}$. The set $X$ will be referred to as an algebraic set approximating $M$. A remarkable theorem of Tognoli (cf. [14, Theorem 14.1.10; 22 , Theorem 7; 27]) asserts that if $M$ is compact and $2 \operatorname{dim} M+1 \leq n$, then $M$ admits an algebraic approximation (cf. also the recent paper of Akbulut and King [8], where the assumption $2 \operatorname{dim} M+1 \leq n$ is relaxed; Tognoli [29] claims a result stronger than [8], but some steps in his argument are incorrect). Several refinements of Tognoli's theorem are known, where the algebraic sets approximating $M$ satisfy certain additional conditions (cf. $[1,2,4,10,11,12$, $22,28]$, where also some important applications are discussed).

In this paper we prove Tognoli-type approximation theorems with precise control of algebraic cycles on algebraic sets approximating $M$. Before stating our results, we need to recall a few notions. For background material the reader may consult the book [14].

Given an affine real algebraic variety $X$, denote by $H_{k}^{\mathrm{alg}}(X, \mathbb{Z} / 2)$ the subgroup of $H_{k}(X, \mathbb{Z} / 2)$ of the homology classes represented by the compact, Zariski closed, $k$-dimensional algebraic subvarieties of $X$. (Cf. [14, Chapter $11 ; 17]$, and also [5, $\S 6 ; 13$, Proposition 2.3]. Another, purely algebraic,

Received by the editors November 28, 1989 and, in revised form, February 22, 1991.

1991 Mathematics Subject Classification. Primary 57R19, 14G30, 14 C99.

The second author's research was supported by NSF Grant DMS- 8905538 . 
description of $H_{k}^{\mathrm{alg}}(X, \mathbb{Z} / 2)$ is given in [21].) Assuming that $X$ is compact and nonsingular, let $H_{k}^{\text {alg }}(X, \mathbb{Z} / 2)$ denote the image of $H_{d-k}^{\text {alg }}(X, \mathbb{Z} / 2)$ under the Poincaré duality isomorphism $H_{d-k}(X, \mathbb{Z} / 2) \rightarrow H^{k}(X, \mathbb{Z} / 2), d=$ $\operatorname{dim} X$. If $\varphi: X \rightarrow Y$ is a regular mapping, then the induced homomorphism $\varphi^{*}: H^{*}(Y, \mathbb{Z} / 2) \rightarrow H^{*}(X, \mathbb{Z} / 2)$ maps $H_{\mathrm{alg}}^{k}(Y, \mathbb{Z} / 2)$ into $H_{\mathrm{alg}}^{k}(X, \mathbb{Z} / 2)$ (cf. [5, Theorem 6.1] or [17]). It is known that $w_{k}(X)$ belongs to $H_{\mathrm{alg}}^{k}(X, \mathbb{Z} / 2)$ for $k \geq 0$, where $w_{k}(X)$ stands for the $k$ th Stiefel-Whitney class of $X[13$, Theorem 2.4; 17, 25].

The important role played by the groups $H_{\mathrm{alg}}^{1}$ in real algebraic geometry is briefly described in the introduction to [15], where the behavior of $H_{\mathrm{alg}}^{1}$ is extensively investigated. Below we also make some comments about $H_{\mathrm{alg}}^{k}$ with $k \geq 2$. Our main result concerns $H_{\mathrm{alg}}^{2}$.

For convenience, we first give the following.

Definition 1.1. Let $M$ be a compact $C^{\infty}$ submanifold of $\mathbb{R}^{n}$ and let $G$ be a subgroup of $H^{k}(M, \mathbb{Z} / 2)$. Then $M$ is said to admit an algebraic approximation of type $G$ if there exists a $C^{\infty}$ embedding $e: M \rightarrow \mathbb{R}^{n}$, arbitrarily close in the $C^{\infty}$ topology to the inclusion mapping $M \hookrightarrow \mathbb{R}^{n}$, such that $X=e(M)$ is a nonsingular algebraic subset of $\mathbb{R}^{n}$ and $h^{*}(G)=H_{\mathrm{alg}}^{k}(X, \mathbb{Z} / 2)$, where $h: X \rightarrow M$ is the diffeomorphism defined by $h(e(m))=m$ for $m$ in $M$.

Proposition 1.2. Let $M$ be a compact $C^{\infty}$ submanifold of $\mathbb{R}^{n}$ with $2 \operatorname{dim} M+1$ $\leq n$. Let $G$ be a subgroup of $H^{k}(M, \mathbb{Z} / 2)$. Then the following conditions are equivalent:

(a) $M$ admits an algebraic approximation of type $G$.

(b) $M$ has an algebraic model of type $G$, that is, one can find an affine nonsingular real algebraic variety $Y$ and a $C^{\infty}$ diffeomorphism $\varphi: Y \rightarrow M$ satisfying $\varphi^{*}(G)=H_{\mathrm{alg}}^{k}(Y, \mathbb{Z} / 2)$.

Proposition 1.2 allows us to restate most of the results of [15] concerning the existence of algebraic models of type $G$, with $G \subset H^{1}$, as theorems on algebraic approximation of type $G$. For instance, we have the following.

Theorem 1.3. Let $M$ be a compact connected $C^{\infty}$ submanifold of $\mathbb{R}^{n}$ of dimension $m$ with $m \geq 3$ and $2 m+1 \leq n$. Let $G$ be a subgroup of $H^{1}(M, \mathbb{Z} / 2)$. Then the following conditions are equivalent:

(a) $M$ admits an algebraic approximation of type $G$.

(b) There exist an affine nonsingular real algebraic variety $X$ and a homeomorphism $\varphi: X \rightarrow M$ such that $\varphi^{*}(G)=H_{\mathrm{alg}}^{1}(X, \mathbb{Z} / 2)$.

(c) $w_{1}(M) \in G$.

Proof. (a) $\Rightarrow$ (b) and (b) $\Rightarrow$ (c) are obvious. If (c) holds, then by [15, Theorems 1.2 and 1.3], $M$ has an algebraic model of type $G$. In virtue of Proposition 1.2, this implies (a).

A slightly weaker version of Theorem 1.3 is valid also in dimension 2 (cf. [15, Theorem 1.4]).

Now let us turn to the case $H_{\mathrm{alg}}^{k}$ with $k \geq 2$. Akbulut and King [1, 2, 4, 23] conjectured that every compact $C^{\infty}$ manifold $M$ is diffeomorphic to an affine nonsingular real algebraic variety $X$ with totally algebraic homology, that is, a 
variety satisfying $H_{k}^{\mathrm{alg}}(X, \mathbb{Z} / 2)=H_{k}(X, \mathbb{Z} / 2)$ for $k \geq 0$. They also described how this conjecture would allow us to simplify several of their proofs and how it would be useful in further work on a topological characterization of real algebraic sets. An interesting counterexample to the conjecture was obtained by Benedetti and Dedò [9]. Our main result can be viewed, in particular, as a systematic study of the phenomenon discovered by them. First let us describe what Benedetti and Dedò have done.

Given a topological space $M$, define $W^{2}(M)$ to be the subset of $H^{2}(M, \mathbb{Z} / 2)$ consisting of the elements of the form $w_{2}(\xi)$, where $\xi$ is a topological real vector bundle over $M$ and $w_{2}(\xi)$ denotes its second Stiefel-Whitney class.

Proposition 1.4. (i) If $M$ is a paracompact topological space, then $W^{2}(M)$ is a subgroup of $H^{2}(M, \mathbb{Z} / 2)$ and every element of $W^{2}(M)$ is of the form $w_{2}(\eta)$ for some topological real vector bundle $\eta$ over $M$ with $w_{1}(\eta)=0$.

(ii) If $X$ is a compact affine nonsingular real algebraic variety, then $H_{\mathrm{alg}}^{2}(X, \mathbb{Z} / 2)$ is a subgroup of $W^{2}(X)$.

Proof. (i) Let $u$ be an element of $W^{2}(M)$ and let $\xi$ be a topological real vector bundle over $M$ with $u=w_{2}(\xi)$. Since $M$ is paracompact, there exists a topological line bundle $\gamma$ over $M$ satisfying $w_{1}(\gamma)=w_{1}(\xi)$ (cf. [20, Chapter 16, Theorem 3.4]). Setting $\eta=\xi \oplus \gamma \oplus \gamma \oplus \gamma$, we obtain $u=w_{2}(\eta)$ and $w_{1}(\eta)=0$. Now it is also obvious that $W^{2}(M)$ is a subgroup of $H^{2}(M, \mathbb{Z} / 2)$.

(ii) This is a consequence of Grothendieck's formula and is contained in [16, Theorem 1.1]. The proof of (ii) given in [9] is not quite correct. At the time when [9] was written, there was no good reference for Grothendieck's formula for varieties defined over $\mathbb{R}$ and the sketch of proof given in [9] has some faults. It assumes the exactness of the global section functor for the so-called $A$-coherent sheaves, which does not hold true. The paper [16] uses an excellent, now available, reference [19, Example 15.3.6].

Counterexample 1.5 [9]. For every positive integer $m$ with $m \geq 11$, there exists a compact, connected, $C^{\infty}$ manifold $M$ of dimension $m$ such that $W^{2}(M) \neq H^{2}(M, \mathbb{Z} / 2)$. The manifold $M$ is constructed by taking the double of a compact, connected, $C^{\infty}$ submanifold (with boundary) $P$ of $\mathbb{R}^{n}$ of dimension $m$ such that the interior of $P$ contains a 5-skeleton $A$ of the EilenbergMac Lane space $K(\mathbb{Z} / 2,2)$ and $A$ is a retract of $P$. In particular, $M$ is orientable (this observation is interesting in view of Theorem 1.7 below). By Proposition 1.4(ii), for every affine nonsingular real algebraic variety $X$ homeomorphic to $M$, one has $H_{\mathrm{alg}}^{2}(X, \mathbb{Z} / 2) \neq H^{2}(X, \mathbb{Z} / 2)$ and hence $X$ does not have totally algebraic homology.

Observe that if $A$ could be embedded in some $C^{\infty}$ manifold $R$ of dimension $k, k<11$, then the above construction would yield an example of a compact $C^{\infty}$ manifold $M$ of dimension $k$ with $H_{\mathrm{alg}}^{2}(X, \mathbb{Z} / 2) \neq H^{2}(X, \mathbb{Z} / 2)$ for each nonsingular real algebraic variety $X$ homeomorphic to $M$. We do not know whether such a manifold $R$ exists.

Remark 1.6. (i) By [26, Theorem II.26], every compact $C^{\infty}$ manifold $M$ with $\operatorname{dim} M \leq 5$ has its homology groups $H_{k}(M, \mathbb{Z} / 2), k \geq 0$, generated by the homology classes represented by compact $C^{\infty}$ submanifolds of $M$. It follows from [2, Theorem 1.1] or [12, Proposition 1] that $M$ has an algebraic model 
with totally algebraic homology. Hence the conjecture of Akbulut and King holds true for manifolds of dimension less than or equal to 5 .

(ii) Akbulut and King [7] proved that every compact $C^{\infty}$ manifold $M$ is homeomorphic to an affine real algebraic variety $X$ with $H_{k}^{\text {alg }}(X, \mathbb{Z} / 2)=$ $H_{k}(X, \mathbb{Z} / 2)$ for $k \geq 0$. However the fact that $X$ is, in general, singular restricts applicability of this theorem.

Our main result is the following.

Theorem 1.7. Let $M$ be a compact connected orientable $C^{\infty}$ submanifold of $\mathbb{R}^{n}$ of dimension $m$ with $m \geq 5$ and $2 m+1 \leq n$. Let $G$ be a subgroup of $H^{2}(M, \mathbb{Z} / 2)$. Then the following conditions are equivalent:

(a) $M$ admits an algebraic approximation of type $G$.

(b) There exists an affine nonsingular real algebraic variety $X$ and a homeomorphism $\varphi: X \rightarrow M$ satisfying $\varphi^{*}(G)=H_{\mathrm{alg}}^{2}(X, \mathbb{Z} / 2)$.

(c) $w_{2}(M) \in G$ and $G \subset W^{2}(M)$.

It follows from Proposition 1.4(ii) and Remark 1.6(i) that if $\operatorname{dim} M=5$, then (c) is equivalent to

(d) $w_{2}(M) \in G$.

If $M$ in Theorem 1.7 is not necessarily orientable and $m$ satisfies only $m \geq$ $3,2 m+1 \leq n$, then we conjecture that conditions (a) and (b) are equivalent to

$\left(\mathbf{c}^{\prime}\right) w_{1}(M) \cup w_{1}(M), w_{2}(M) \in G$, and $G \subset W^{2}(M)$.

Of course, $U$ stands for the cup product. Clearly, $\left(\mathrm{c}^{\prime}\right)$ is equivalent to

$\left(\mathbf{d}^{\prime}\right) w_{1}(M) \cup w_{1}(M), w_{2}(M) \in G$

for $\operatorname{dim} M \leq 5$.

No general result is known concerning the algebraic approximation of type $G$ with $G$ contained in $H^{k}(M, \mathbb{Z} / 2), k \geq 3$.

\section{Proofs of Proposition 1.2 AND Theorem 1.7}

Proof of Proposition 1.2. (a) $\Rightarrow$ (b) is obvious, so we only have to show (b) $\Rightarrow$ (a).

Let $Y$ be an affine nonsingular real algebraic variety and let $\varphi: Y \rightarrow M$ be a $C^{\infty}$ diffeomorphism satisfying $\varphi^{*}(G)=H_{\mathrm{alg}}^{k}(Y, \mathbb{Z} / 2)$. Clearly, $\varphi^{-1}: M \rightarrow Y$ is bordant to the identity mapping of $Y$ and hence, by [1, Proposition 4.1] (cf. also [4, 22]), one can find a $C^{\infty}$ embedding $e: M \rightarrow \mathbb{R}^{n}$, arbitrarily close in the $C^{\infty}$ topology to the inclusion mapping $M \hookrightarrow \mathbb{R}^{n}$, such that $X=e(M)$ is a nonsingular algebraic subset of $\mathbb{R}^{n}$ and there exists a regular mapping $\psi: X \rightarrow Y$ with the property that $\psi \circ e$ is close in the $C^{\infty}$ topology to $\varphi^{-1}$ (in particular, we may assume that $\psi$ is a $C^{\infty}$ diffeomorphism).

We claim that $\psi^{*}\left(H_{\mathrm{alg}}^{k}(Y, \mathbb{Z} / 2)\right)=H_{\mathrm{alg}}^{k}(X, \mathbb{Z} / 2)$. Indeed, since $\psi$ is a regular mapping, we obtain $\psi^{*}\left(H_{\mathrm{alg}}^{k}(Y, \mathbb{Z} / 2)\right) \subset H_{\mathrm{alg}}^{k}(X, \mathbb{Z} / 2)$. Now let $u$ be an element of $H_{\mathrm{alg}}^{k}(X, \mathbb{Z} / 2)$. By definition, $u=D_{X}^{-1}([V])$, where $D_{X}: H^{*}(X, \mathbb{Z} / 2) \rightarrow$ $H_{*}(X, \mathbb{Z} / 2)$ is the Poincaré duality isomorphism and $[V]$ is the homology class in $H_{*}(X, \mathbb{Z} / 2)$ represented by a Zariski closed algebraic subvariety $V$ of $X$ of dimension $\operatorname{dim} X-k$. Note that since $\psi$ is a diffeomorphism, we have $\psi_{*} \circ D_{X} \circ \psi^{*}=D_{Y}$, where $\psi_{*}: H_{*}(X, \mathbb{Z} / 2) \rightarrow H_{*}(Y, \mathbb{Z} / 2)$ is the homomorphism induced by $\psi$ and $D_{Y}: H^{*}(Y, \mathbb{Z} / 2) \rightarrow H_{*}(Y, \mathbb{Z} / 2)$ is the Poincaré 
duality isomorphism. It follows that

$$
\left(\psi^{*}\right)^{-1}(u)=\left(D_{Y}^{-1} \circ \psi_{*} \circ D_{X}\right)(u)=D_{Y}^{-1}\left(\psi_{*}([V])\right) .
$$

On the other hand, by $[14$, Lemma 11.3 .4$]$, we obtain $\psi_{*}([V])=[W]$, where $W$ is the Zariski closure of $\psi(V)$ in $Y$, and hence the claim is proved.

By construction, if $h: X \rightarrow M$ is the diffeomorphism defined by $h(e(m))=$ $m$ for $m$ in $M$, then $h^{*}(G)=H_{\text {alg }}^{k}(X, \mathbb{Z} / 2)$. This completes the proof of the proposition.

For the convenience of the reader, we now collect a few results that will be used in the proof of Theorem 1.7.

Let $X$ be an affine real algebraic variety. An algebraic (real) vector bundle over $X$ is said to be strongly algebraic if it is algebraically isomorphic to a subbundle of a product vector bundle $X \times \mathbb{R}^{n}$ for some $n$ (cf. [14, Chapter 12] for an exposition of the theory of strongly algebraic vector bundles). We shall say that a topological (real) vector bundle over $X$ admits an algebraic structure if it is topologically isomorphic to a strongly algebraic vector bundle over $X$.

Theorem 2.1. Let $X$ be a compact affine nonsingular real algebraic variety with $\operatorname{dim} X=2$ or 3 . Let $\xi$ be a topological vector bundle over $X$ of constant rank and let $C$ be a compact, connected, $C^{\infty}$ curve in $X$ Then:

(i) The vector bundle $\xi$ admits an algebraic structure if and only if $w_{k}(\xi)$ belongs to $H_{\mathrm{alg}}^{k}(X, \mathbb{Z} / 2)$ for $k=1,2$.

(ii) If the homology class in $H_{1}(X, \mathbb{Z} / 2)$ represented by $C$ belongs to $H_{1}^{\mathrm{alg}}(X, \mathbb{Z} / 2)$, then there exists a $C^{\infty}$ embedding $e: C \rightarrow X$, arbitrarily close in the $C^{\infty}$ topology to the inclusion mapping $C \hookrightarrow X$, such that $e(C)$ is a nonsingular, Zariski closed, algebraic subvariety of $X$.

Proof. For $\operatorname{dim} X=2$, Theorem 2.1 is well known (cf. [14, Theorem 12.5.3]). If $\operatorname{dim} X=3$, then (i) is proved in [16, Theorem 1.6], while (ii) is equivalent to [16, Corollary 1.8] combined with [6, Proposition 2].

Denote by $G_{p, q}$ the Grassmann variety of $q$-dimensional vector subspaces of $\mathbb{R}^{p}$. Recall that $G_{p, q}$ has the structure of an affine nonsingular real algebraic variety [14, Theorem 3.4.4 and Proposition 3.4.3] and the universal vector bundle $\gamma_{p, q}$ over $G_{p, q}$ is strongly algebraic [14, Proposition 12.1.8].

Theorem 2.2. Let $X$ be a compact affine nonsingular real algebraic variety and let $f: X \rightarrow G_{p, q}$ be a $C^{\infty}$ mapping. Then the following conditions are equivalent:

(a) $f$ can be approximated in the $C^{\infty}$ topology by regular mappings from $X$ to $G_{p, q}$.

(b) The pull-back vector bundle $f^{*} \gamma_{p, q}$ admits an algebraic structure.

Proof. [14, Theorem 13.3.1] or [22, Lemma 14].

Theorem 2.3. Let $M$ be a compact $C^{\infty}$ submanifold of $\mathbb{R}^{n}$ of dimension $m$ with $2 m+1 \leq n$. Let $Y$ be a $C^{\infty}$ submanifold of $M$ which is also a nonsingular algebraic subset of $\mathbb{R}^{n}$. Let $V=G_{p_{1}, q_{1}} \times \cdots \times G_{p_{s}, q_{s}}$ and let $f: M \rightarrow V$ be a $C^{\infty}$ mapping. Assume that the restriction $f \mid Y$ is a regular mapping and the restriction $\nu \mid Y$ of the normal vector bundle $\nu$ of $M$ in $\mathbb{R}^{n}$ to $Y$ admits an algebraic structure. Then one can find a $C^{\infty}$ embedding $e: M \rightarrow \mathbb{R}^{n}, a$ nonsingular algebraic subset $X$ of $\mathbb{R}^{n}$, and a regular mapping $g: X \rightarrow V$ such 
that $X=e(M), e$ (resp. $g \circ e$ ) is arbitrarily close in the $C^{\infty}$ topology to the inclusion mapping $M \hookrightarrow \mathbb{R}^{n}$ (resp. $f$ ), and $e(x)=x$ for $x$ in $Y$.

Proof. This is just a minor modification of results proved by Akbulut and King [1], and Benedetti and Tognoli [10, 12, 28].

Indeed, if $\varphi: Y \rightarrow G_{n, n-m}$ is the $C^{\infty}$ mapping defined by

$$
\varphi(y)=\text { the orthogonal complement of } T_{y} M \text { in } \mathbb{R}^{n}
$$

for $y$ in $Y$, where $T_{y} M$ is the tangent space of $M$ at $y$, then the pull-back vector bundle $\varphi^{*} \gamma_{n, n-m}$ is isomorphic to $\nu \mid Y$ and hence, in virtue of Theorem 2.2, $\varphi$ can be approximated in the $C^{\infty}$ topology by regular mappings. Now Theorem 2.3 follows immediately from [12, Theorem 3] (cf. also [1, Proposition 2.8, Lemma 2.7; 10, 28]).

Lemma 2.4. Let $S$ be a compact connected nonorientable $C^{\infty}$ surface of even genus and let $S^{1}$ be the unit circle. Let $\varphi: S \times S^{1} \rightarrow \mathbb{R}^{n}, n \geq 7$, be a $C^{\infty}$ embedding. Set $N=\varphi\left(S \times S^{1}\right)$ and $S_{0}=\varphi\left(S \times\left\{y_{0}\right\}\right)$, where $y_{0}$ is in $S^{1}$. Let $\sigma: S_{0} \hookrightarrow N$ be the inclusion mapping and let $G=\operatorname{Ker} \sigma^{*}$ be the kernel of the induced homomorphism $\sigma^{*}: H^{2}(N, \mathbb{Z} / 2) \rightarrow H^{2}\left(S_{0}, \mathbb{Z} / 2\right)$. Then $N$ admits an algebraic approximation of type $G$.

Proof. By [2, Theorem 1.1] or [12, Proposition 1], one can find a nonsingular real algebraic subset $V$ of $\mathbb{R}^{p}$, for some $p \geq 7$, with the property that $V$ is diffeomorphic to $S$ and $H_{1}(V, \mathbb{Z} / 2)$ is generated by the homology classes of nonsingular algebraic curves $D_{1}, \ldots, D_{k}$ in $V$. In particular, $H_{1}^{\text {alg }}(V, \mathbb{Z} / 2)=$ $H_{1}(V, \mathbb{Z} / 2)$ and hence, by Theorem 2.1 (i) every topological vector bundle over $V$ admits an algebraic structure.

Let $P$ be a compact $C^{\infty}$ manifold with boundary $\partial P$ equal to $S$ ( $P$ exists since $S$ is of even genus). Let $Q$ be the double of $P$. Since $p \geq 7$, there exists a $C^{\infty}$ embedding $F: Q \rightarrow \mathbb{R}^{p}$ with $F(S)=V$. By Theorem 2.3, we may assume that $W=F(Q)$ is a nonsingular algebraic subset of $\mathbb{R}^{p}$.

Let $C$ be the algebraic curve in $\mathbb{R}^{2}$ defined by the equation

$$
x^{4}+y^{4}-4 x^{2}+1=0 \text {. }
$$

Then $C$ is nonsingular, irreducible, and has two connected components, say $C_{1}$ and $C_{2}$, diffeomorphic to $S^{1}$. Let $p$ be a point in $V$ and $E=\{p\} \times C_{1}$.

We claim that there exists a neighborhood $\mathscr{U}$ (in the $C^{\infty}$ topology) of the inclusion mapping $E \hookrightarrow W \times C$ such that $e(E)$ is not a nonsingular algebraic subvariety of $W \times C$ for $e$ in $\mathscr{U}$. Indeed, suppose for a moment that such a neighborhood $\mathscr{U}$ does not exist. Then one can find a $C^{\infty}$ embedding $e: E \rightarrow$ $W \times C$ such that $e(E)$ is a nonsingular algebraic subvariety of $W \times C$ and the canonical projection $\pi: W \times C \rightarrow C$ maps $e(E)$ diffeomorphically onto $C_{1}$. However, since $\pi$ is a regular mapping, it follows from [14, Lemma 11.3.4] that $\operatorname{dim}(C \backslash \pi(e(E)))=0$, a contradiction. Hence the claim is proved.

By construction, $V \times C_{1}$ bounds in $W \times C$ and therefore there exists a $C^{\infty}$ function $f: W \times C \rightarrow \mathbb{R}$ such that 0 in $\mathbb{R}$ is its regular value and $V \times C_{1}=f^{-1}(0)$. Let $c$ be a point in $C_{1}$. Then one can find a regular function $g: W \times C \rightarrow \mathbb{R}$, close in the $C^{\infty}$ topology to $f$ and vanishing on the $D_{i} \times\{c\}, i=1, \ldots, k$ (cf. [28, p. 75]). If $g$ is sufficiently close to $f$, then $X=g^{-1}(0)$ is a nonsingular algebraic subvariety of $W \times C$ diffeomorphic to $N$ and there exists an embedding $e_{0}$ in $\mathscr{U}$ with $e_{0}(E)$ contained in $X$. It follows 
that if $\varepsilon: e_{0}(E) \rightarrow X$ is a $C^{\infty}$ embedding sufficiently close in the $C^{\infty}$ topology to the inclusion mapping $e_{0}(E) \hookrightarrow X$, then $\varepsilon\left(e_{0}(E)\right)$ is not a nonsingular algebraic subvariety of $X$. By Theorem 2.1 (ii), the homology class in $H_{1}(X, \mathbb{Z} / 2$ ) represented by $e_{0}(E)$ does not belong to $H_{1}^{\text {alg }}(X, \mathbb{Z} / 2)$. Hence $H_{1}^{\text {alg }}(X, \mathbb{Z} / 2)$ is generated by the homology classes of the $D_{i} \times\{c\}, i=1, \ldots, k$. In other words, $N$ has an algebraic model of type $G$. By Proposition 1.2, $N$ admits an algebraic approximation of type $G$.

We also need one more, purely topological, observation.

Lemma 2.5. Let $M$ be a compact connected $C^{\infty}$ manifold with $\operatorname{dim} M \geq 4$. Then every homology class in $H_{2}(M, \mathbb{Z} / 2)$ can be represented by a compact connected nonorientable $C^{\infty}$ surface in $M$ of even topological genus.

Proof. Let $u$ be an element of $H_{2}(M, \mathbb{Z} / 2)$ and $m=\operatorname{dim} M$. By [26, Theorem II.26], there exists a compact $C^{\infty}$ surface $S$ in $M$ representing $u$. Let $D_{1}$ and $D_{2}$ be disjoint $m$-dimensional disks in $M$ which are also disjoint from $S$. Let $P_{i}$ be a $C^{\infty}$ surface in $D_{i}$ diffeomorphic to the real projective plane, $i=1,2$. Replacing $S$ by a connected sum in $M$ of the connected components of $S$ and $P_{1}$, we may assume that $S$ is connected and nonorientable. If $S$ has even topological genus, we are done. Otherwise, it suffices to replace $S$ by a connected sum in $M$ of $S$ and $P_{2}$.

Proof of Theorem 1.7. (a) $\Rightarrow$ (b) is obvious, while (b) $\Rightarrow$ (c) follows from the fact that $w_{2}(X)$ is in $H_{\mathrm{alg}}^{2}(X, \mathbb{Z} / 2)$ and Proposition 1.4(ii). Thus it suffices to show $(\mathrm{c}) \Rightarrow(\mathrm{a})$.

Given a subset $A$ of $M$, we shall denote by $r_{A}: H^{*}(M, \mathbb{Z} / 2) \rightarrow H^{*}(A, \mathbb{Z} / 2)$ the homomorphism induced by the inclusion mapping $A \hookrightarrow M$.

Let $H$ be a subgroup of $H^{2}(M, \mathbb{Z} / 2)$ such that $G \oplus H=H^{2}(M, \mathbb{Z} / 2)$ and let $\left(v_{1}, \ldots, v_{k}\right)$ be a $\mathbb{Z} / 2$-basis for $H$. Since the bilinear mapping

$$
H^{2}(M, \mathbb{Z} / 2) \times H^{m-2}(M, \mathbb{Z} / 2) \rightarrow H^{m}(M, \mathbb{Z} / 2), \quad(v, w) \rightarrow v \cup w,
$$

where $U$ stands for the cup product, is a dual pairing (cf. [18, Proposition 8.13]), it follows that there exist elements $a_{1}, \ldots, a_{k}$ in $H^{m-2}(M, \mathbb{Z} / 2)$ with the properties that $u \cup a_{i}=0, v_{j} \cup a_{i}=0$, and $v_{i} \cup a_{i} \neq 0$ for $u$ in $G$, $i, j=1, \ldots, k, i \neq j$. Let $\alpha_{i}$ be the homology class in $H_{2}(M, \mathbb{Z} / 2)$ Poincaré dual to $a_{i}$. By Lemma 2.5 , one can find a compact connected $C^{\infty}$ surface $S_{i}$ in $M$ representing $\alpha_{i}$, which is nonorientable of even topological genus. Since $m \geq 5$, we may assume that the surfaces $S_{i}$ are mutually disjoint. A standard calculation involving the cup and cap products shows that given $u$ in $H^{2}(M, \mathbb{Z} / 2)$, one has

$$
r_{S_{i}}(u)=0 \text { for } i=1, \ldots, k \text { if and only if } u \in G \text {. }
$$

Similarly, one also shows

$$
r_{S_{i}}\left(v_{i}\right) \neq 0
$$

Let $U_{i}$ be a small tubular neighborhood of $S_{i}$ in $M$ (in particular, the $U_{i}$ are mutually disjoint, $i=1, \ldots, k)$. Shrinking $U_{i}$ if necessary, we obtain from (1) that for $u$ in $H^{2}(M, \mathbb{Z} / 2)$,

$$
r_{U_{i}}(u)=0 \text { for } i=1, \ldots, k \text { if and only if } u \in G \text {. }
$$


We claim that there exist a $C^{\infty}$ submanifold $Y_{i}$ of $U_{i}$ containing $S_{i}$ and a $C^{\infty}$ diffeomorphism $\varphi_{i}: S_{i} \times S^{1} \rightarrow Y_{i}$ satisfying $S_{i}=\varphi_{i}\left(S_{i} \times\left\{y_{0}\right\}\right)$, where $S^{1}$ is the unit circle and $y_{0}$ is a point in $S^{1}$. Indeed, let us identify $U_{i}$ with the total space of the normal vector bundle $\nu_{i}$ of $S_{i}$ in $M$. We have $\nu_{i} \oplus \tau\left(S_{i}\right)=\tau(M) \mid S_{i}$, where $\tau(\cdot)$ stands for the tangent bundle. Since $M$ is orientable, $w_{1}(\tau(M))=0$ and hence $w_{1}\left(\nu_{i}\right)=w_{1}\left(\tau\left(S_{i}\right)\right)$. By (c) and (1), $w_{2}\left(\tau(M) \mid S_{i}\right)=r_{S_{i}}\left(w_{2}(M)\right)=0$. It follows that

$$
\begin{aligned}
w_{2}\left(\nu_{i}\right) & =w_{2}\left(\tau\left(S_{i}\right)\right)+w_{1}\left(\nu_{i}\right) \cup w_{1}\left(\tau\left(S_{i}\right)\right) \\
& =w_{2}\left(\tau\left(S_{i}\right)\right)+w_{1}\left(\tau\left(S_{i}\right)\right) \cup w_{1}\left(\tau\left(S_{i}\right)\right) .
\end{aligned}
$$

Since $S_{i}$ is nonorientable of even topological genus, we have $w_{2}\left(\tau\left(S_{i}\right)\right)=0$ and $w_{1}\left(\tau\left(S_{i}\right)\right) \cup w_{1}\left(\tau\left(S_{i}\right)\right)=0$, and hence $w_{2}\left(\nu_{i}\right)=0$. Let $\zeta_{i}$ be a real line bundle over $S_{i}$ with $w_{1}\left(\zeta_{i}\right)=w_{1}\left(\nu_{i}\right)$. It follows (cf., for example, [16, Remark 1.4]) that $\nu_{i}$ and $\zeta_{i}$ are stably equivalent. Since $\operatorname{dim} S_{i}=2$ and $\operatorname{rank} \nu_{i}=m-2$, this implies that $\nu_{i}$ is isomorphic to the direct sum of $\zeta_{i}$ and a trivial vector bundle of rank $m-3$ (cf. [20, Chapter 8, Theorem 1.5]). It follows that $S_{i} \subset \widetilde{S}_{i} \subset U_{i}$, where $\widetilde{S}_{i}$ is diffeomorphic to $S_{i} \times \mathbb{R}^{2}$. Now the claim is obvious.

It follows from (3) that given $u$ in $H^{2}(M, \mathbb{Z} / 2)$, we have

$$
r_{Y_{i}}(u)=0 \text { for } i=1, \ldots, k \text { if and only if } u \in G \text {. }
$$

Also note that $(2)$ implies

$$
r_{Y_{i}}\left(v_{i}\right) \notin \operatorname{Ker} \sigma_{i}^{*} \quad \text { for } i=1, \ldots, k,
$$

where $\sigma_{i}: S_{i} \hookrightarrow Y_{i}$ is the inclusion mapping.

By Lemma 2.4, there exists a $C^{\infty}$ embedding $e_{i}: Y_{i} \rightarrow \mathbb{R}^{n}$, arbitrarily close in the $C^{\infty}$ topology to the inclusion mapping $Y_{i} \hookrightarrow \mathbb{R}^{n}$, such that $e_{i}\left(Y_{i}\right)$ is a nonsingular algebraic subset of $\mathbb{R}^{n}$ and $H_{\mathrm{alg}}^{2}\left(e_{i}\left(Y_{i}\right), \mathbb{Z} / 2\right)=\operatorname{Ker} s_{i}^{*}$, where $s_{i}: e_{i}\left(S_{i}\right) \hookrightarrow e_{i}\left(Y_{i}\right)$ is the inclusion mapping. Now one can find a $C^{\infty}$ embedding $E: M \rightarrow \mathbb{R}^{n}$, close in the $C^{\infty}$ topology to the inclusion mapping $M \hookrightarrow \mathbb{R}^{n}$, satisfying $E \mid Y_{i}=e_{i}$ for $i=1, \ldots, k$. Hence, replacing possibly $M, Y_{i}$, and $S_{i}$ by $E(M), E\left(Y_{i}\right)$, and $E\left(S_{i}\right)$, respectively, we may assume that $Y_{i}$ is a nonsingular algebraic subset of $\mathbb{R}^{n}$ and

$$
H_{\text {alg }}^{2}\left(Y_{i}, \mathbb{Z} / 2\right)=\operatorname{Ker} \sigma_{i}^{*} \quad \text { for } i=1, \ldots, k .
$$

By (5) and (6), we obtain

$$
r_{Y_{i}}\left(v_{i}\right) \in H^{2}\left(Y_{i}, \mathbb{Z} / 2\right) \backslash H_{\mathrm{alg}}^{2}\left(Y_{i}, \mathbb{Z} / 2\right) \text { for } i=1, \ldots, k .
$$

Let $G=\left\{u_{1}, \ldots, u_{l}\right\}$. By (c) and Proposition 1.4(i), $u_{j}=w_{2}\left(\xi_{j}\right)$ for some topological real vector bundle $\xi_{j}$ over $M$ with $w_{1}\left(\xi_{j}\right)=0$ for $j=$ $1, \ldots, l$. Let $f_{j}: M \rightarrow G_{p_{j}, q_{j}}$ be a $C^{\infty}$ classifying mapping for $\xi_{j}$, that is, $\xi_{j}$ is isomorphic to the pull-back vector bundle $f_{j}^{*} \gamma_{p_{j}, q_{j}}$.

Clearly, $Y=Y_{1} \cup \cdots \cup Y_{k}$ is a $C^{\infty}$ submanifold of $M$ which is a nonsingular algebraic subset of $\mathbb{R}^{n}$. We have, obviously, $w_{1}\left(\xi_{j} \mid Y\right)=0$ and, by (4), $w_{2}\left(\xi_{j} \mid Y\right)=0$. Hence, in virtue of Theorem 2.1(i), the vector bundle $\xi_{j} \mid Y \cong\left(f_{j} \mid Y\right)^{*} \gamma_{p_{j}, q_{j}}$ over $Y$ admits an algebraic structure. Now, by Theorem $2.2, f_{j} \mid Y$ can be approximated in the $C^{\infty}$ topology by regular mappings from $Y$ to $G_{p_{j}, q_{j}}$. Using this fact, and possibly modifying $f_{j}$ without changing its homotopy type, we may assume that $f_{j} \mid Y$ is a regular mapping. Set $f=\left(f_{1}, \ldots, f_{l}\right)$ and $V=G_{p_{1}, q_{1}} \times \cdots \times G_{p_{l}, q_{l}}$. 
Let $\nu$ be the normal vector bundle of $M$ in $\mathbb{R}^{n}$. Since $M$ is orientable, $w_{1}(\nu)=0$ and hence $w_{1}(\nu \mid Y)=0$. By $(\mathrm{c}), w_{2}(\nu)=w_{2}(M)$ is in $G$ and therefore, in virtue of $(4), w_{2}(\nu \mid Y)=0$. Applying Theorem 2.1(i), we conclude that $\nu \mid Y$ admits an algebraic structure.

Now, the properties of $M, Y, f$, and $\nu$ established above allow us to apply Theorem 2.3. Thus one can find a $C^{\infty}$ embedding $e: M \rightarrow \mathbb{R}^{n}$, arbitrarily close in the $C^{\infty}$ topology to the inclusion mapping $M \hookrightarrow \mathbb{R}^{n}$, such that $X=e(M)$ is a nonsingular algebraic subset of $\mathbb{R}^{n}, e(x)=x$ for $x$ in $Y$, and there exists a regular mapping $g=\left(g_{1}, \ldots, g_{l}\right): X \rightarrow V$ with $g \circ e$ close to $f$ in the $C^{\infty}$ topology (in particular, we may assume that $g \circ e$ is homotopic to $f$ ).

Let $h: X \rightarrow M$ be the $C^{\infty}$ diffeomorphism defined by $h(e(m))=m$ for $m$ in $M$. By construction, $h^{*}(G)$ consists of the elements of the form $w_{2}\left(g_{j}^{*} \gamma_{p_{j}, q_{j}}\right), j=1, \ldots, l$. Since $g_{j}$ is a regular mapping, the vector bundle $g_{j}^{*} \gamma_{p_{j}, q_{j}}$ is strongly algebraic and hence by $[13,17,25], w_{2}\left(g_{j}^{*} \gamma_{p_{j}, q_{j}}\right)$ is in $H_{\mathrm{alg}}^{2}\left(X, \mathbb{Z}_{2}\right)$. Therefore, $h^{*}(G) \subset H_{\mathrm{alg}}^{2}\left(X_{1}, \mathbb{Z} / 2\right)$. Moreover, if $\rho_{i}: H^{*}(X, \mathbb{Z} / 2)$ $\rightarrow H^{*}\left(Y_{i}, \mathbb{Z} / 2\right)$ is the homomorphism induced by the inclusion mapping $Y_{i} \hookrightarrow X$, then $\rho_{i}\left(h^{*}\left(v_{i}\right)\right)=r_{Y_{i}}\left(v_{i}\right)$ and hence by $(7)$, we have $h^{*}(H) \cap$ $H_{\text {alg }}^{2}(X, \mathbb{Z} / 2)=\{0\}$. Now it follows that $H^{*}(G)=H_{\text {alg }}^{2}(X, \mathbb{Z} / 2)$ and the proof of $(\mathrm{c}) \Rightarrow(\mathrm{a})$ is complete.

Added in proof. M. Kreck has informed us that his student P. Teichner recently obtained the following. For each $m \geq 6$ there exists a compact, orientable $C^{\infty}$ manifold $M$ of dimension $m$ such that $H^{2}(M, \mathbb{Z} / 2) \neq W^{2}(M)$. It follows from Proposition 1.4 that each algebraic model $X$ of $M$ has $H_{\text {alg }}^{2}(X, \mathbb{Z} / 2) \neq$ $H^{2}(X, \mathbb{Z} / 2)$.

\section{REFERENCES}

1. S. Akbulut and H. King, The topology of real algebraic sets with isolated singularities, Ann. of Math. (2) 113 (1981), 425-446.

2. __ A relative Nash theorem, Trans. Amer. Math. Soc. 267 (1981), 465-481.

3. __ Real algebraic structures on topological spaces, Inst. Hautes Études Sci. Publ. Math. 53 (1981), 79-167.

4. __ The topology of real algebraic sets, Enseign. Math. 29 (1983), 221-261.

5. __ Submanifolds and homology of nonsingular real algebraic varieties, Amer. J. Math. 107 (1985), 45-83.

6. __ Polynomial equations of immersed surfaces, Pacific J. Math. 131 (1988), 209-217.

7. __ All compact manifolds are homeomorphic to totally algebraic real algebraic sets, Comment. Math. Helv. 66 (1990), 139-149.

8. Invent. Math. 107 (1992), 87-98.

9. R. Benedetti and M. Dedò, Counterexamples to representing homology classes by real algebraic subvarieties up to homeomorphism, Compositio Math. 53 (1984), 143-151.

10. R. Benedetti and A. Tognoli, Approximation theorems in real algebraic geometry, Algebra é Geometria, Supl. Boll. Un. Mat. Ital. 2 (1980), 209-228.

11. __, On real algebraic vector bundles, Bull. Sci. Math. (2) 104 (1980), 89-112.

12. __ Théorèmes d'approximation en géométrie algébrique réelle, Séminaire sur la Géométrie Algébrique Réelle, Publ. Math. Univ. Paris VII, vol. 9, Univ. Paris VII, Paris, 1980, pp. 123-145. 
13. - Remarks and counterexamples in the theory of real algebraic vector bundles and cycles, Géométrie Algébrique Réelle et Formes Quadratiques, Lecture Notes in Math., vol. 959, Springer, Berlin and New York, 1982, pp. 198-211.

14. J. Bochnak, M. Coste, and M.-F. Roy, Géométrie algébrique réelle, Ergeb. Math. Grenzgeb. (3), vol. 12, Springer, Berlin and New York, 1987.

15. J. Bochnak and W. Kucharz, Algebraic models of smooth manifolds, Invent. Math. 97 (1989), 585-611.

16.,$K$-theory of real algebraic surfaces and threefolds, Math. Proc. Cambridge Philos. Soc. 106 (1989), 471-480.

17. A. Borel and A. Haefliger, La classe d'homologie fondamentale d'un espace analytique, Bull. Soc. Math. France 89 (1961), 461-513.

18. A. Dold, Lectures on algebraic topology, Grundlehren Math. Wiss., vol. 200, Springer, Berlin and New York, 1980.

19. W. Fulton, Intersection theory, Ergeb. Math. Grenzgeb. (3), vol. 2, Springer, Berlin and New York, 1984.

20. D. Husemoller, Fibre bundles, Springer, Berlin and New York, 1975.

21. F. Ischebeck and H.-W. Schülting, Rational and homological equivalence of real cycles, Invent. Math. 94 (1988), 307-316.

22. N. Ivanov, Approximation of smooth manifolds by real algebraic sets, Russian Math. Surveys 37 (1982), no. 1, 1-59.

23. H. King, Survey on the topology of real algebraic sets, Rocky Mountain J. Math. 14 (1984), 821-830.

24. W. Kucharz, On homology of real algebraic sets, Invent. Math. 82 (1985), 19-26.

25. M. Shiota, Real algebraic realizations of characteristic classes, Publ. Res. Inst. Math. Sci. Kyoto Univ. 18 (1982), 995-1008.

26. R. Thom, Quelques propriétés globales des variétés différentiables, Comment. Math. Helv. 28 (1954), 17-86.

27. A. Tognoli, Su una congettura di Nash, Ann. Scuola Norm. Sup. Pisa 27 (1973), 167-185.

28. __ Algebraic approximation of manifolds and spaces (Séminaire Bourbaki 32e année, 1979/1980, no. 548), Lecture Notes in Math., vol. 842, Springer, Berlin and New York, 1981, pp. 73-94.

29. __ Any compact differential submanifold of $\mathbb{R}^{n}$ has an algebraic approximation in $\mathbb{R}^{n}$, Topology 27 (1988), 205-210.

Department of Mathematics, Vrije Universiteit, P. O. Box 7161, MC 1007 Amsterdam, THE NETHERLANDS

Department of Mathematics, University of Hawail at Manoa, 2565 The Mall, HonOLULU, HaWAII 96822

Current address: Department of Mathematics and Statistics, University of New Mexico, Albuquerque, New Mexico 87131-1141 